\title{
Photospheric activity, rotation, and star-planet interaction of the planet-hosting star CoRoT-6*
}

\author{
A. F. Lanza ${ }^{1}$, A. S. Bonomo ${ }^{1,2}$, I. Pagano ${ }^{1}$, G. Leto ${ }^{1}$, S. Messina ${ }^{1}$, G. Cutispoto ${ }^{1}$, C. Moutou ${ }^{2}$, S. Aigrain ${ }^{3}$, R. Alonso ${ }^{4}$,

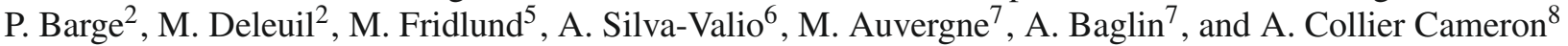 \\ ${ }^{1}$ INAF - Osservatorio Astrofisico di Catania, via S. Sofia, 78, 95123 Catania, Italy \\ e-mail: nuccio.lanza@oact.inaf.it \\ ${ }^{2}$ Laboratoire d'Astrophysique de Marseille (UMR 6110), Technopole de Château-Gombert, 38 rue Frédéric Joliot-Curie, \\ 13388 Marseille Cedex 13, France \\ 3 Department of Physics, University of Oxford, Denys Wilkinson Building, Keble Road, Oxford OX1 3RH, UK \\ 4 Observatoire de Genève, Université de Genève, 51 Ch. des Maillettes, 1290 Sauverny, Switzerland \\ 5 Research and Scientific Support Department, European Space Agency, Keplerlaan 1, 2200AG, Noordwijk, The Netherlands \\ ${ }^{6}$ CRAAM, Mackenzie University, rua de Consolação 896, 01302-907 São Paulo, Brazil \\ 7 LESIA, CNRS UMR 8109, Observatoire de Paris, 5 place J. Janssen, 92195 Meudon, France \\ 8 School of Physics and Astronomy, University of St. Andrews, North Haugh, St Andrews, Fife KY16 9SS, Scotland, UK
}

Received 21 June 2010 / Accepted 16 September 2010

\begin{abstract}
Context. The CoRoT satellite has recently discovered a hot Jupiter that transits across the disc of a F9 main-sequence star called CoRoT-6 with a period of 8.886 days.

Aims. We model the photospheric activity of the star and use the maps of the active regions to study stellar differential rotation and the star-planet interaction.

Methods. We apply a maximum entropy spot model to fit the optical modulation as observed by CoRoT during a uninterrupted interval of $\sim 140$ days. Photospheric active regions are assumed to consist of spots and faculae in a fixed proportion with solar-like contrasts. Results. Individual active regions have lifetimes up to 30-40 days. Most of them form and decay within five active longitudes whose different migration rates are attributed to the stellar differential rotation for which a lower limit of $\Delta \Omega / \Omega=0.12 \pm 0.02$ is obtained. Several active regions show a maximum of activity at a longitude lagging the subplanetary point by $\sim 200^{\circ}$ with the probability of a chance occurrence being smaller than 1 percent.

Conclusions. Our spot modelling indicates that the photospheric activity of CoRoT-6 could be partially modulated by some kind of star-planet magnetic interaction, while an interaction related to tides is highly unlikely because of the weakness of the tidal force.
\end{abstract}

Key words. stars: magnetic field - stars: late-type - stars: activity - stars: rotation - planetary systems - stars: individual: CoRoT-6

\section{Introduction}

CoRoT (Convection, Rotation and Transits) is a photometric space experiment devoted to asteroseismology and the search for extrasolar planets by the method of transits (Baglin et al. 2006). It has recently discovered a hot Jupiter with an orbital period of 8.886 days and a mass of $2.96 \pm 0.34 M_{\mathrm{J}}$ orbiting a F9 mainsequence star dubbed CoRoT-6 (Fridlund et al. 2010). The star is magnetically active and shows a rotational modulation of its optical flux with a relative amplitude of $\sim 2.7$ percent and a period of $\sim 6.4$ days.

We study the photospheric activity of CoRoT-6 by applying the spot modelling approach already used for the high-precision light curves of CoRoT-2 (Lanza et al. 2009a), CoRoT-4 (Lanza et al. 2009b), and CoRoT-7 (Lanza et al. 2010). It allows us to map the longitudinal distribution of photospheric active regions and trace their evolution during the $\sim 140$ days of CoRoT observations. The different rotation rates of active regions allow us to

^ Based on observations obtained with CoRoT, a space project operated by the French Space Agency, CNES, with partecipation of the Science Programme of ESA, ESTEC/RSSD, Austria, Belgium, Brazil, Germany, and Spain. estimate a lower limit for the amplitude of the differential rotation of CoRoT-6. We also use the maps to look for signs of star-planet interaction and find some statistical evidence of active regions lagging the subplanetary point by $\sim 170^{\circ}-200^{\circ}$ during some time intervals. The case for a star-planet interaction in other systems has been recently reviewed by Lanza (2008) and Shkolnik et al. (2009).

\section{Observations}

CoRoT-6 has been observed during the second long run of CoRoT toward the galactic centre from 15 April to 7 September 2008. Since the star is relatively bright $(V=13.91 \mathrm{mag})$, the time sampling is $32 \mathrm{~s}$ from the beginning of the observations. CoRoT performs aperture photometry with a fixed mask (see Fig. 3 in Fridlund et al. 2010). The contaminating flux from background stars falling inside the mask amounts to $2.8 \pm$ 0.7 percent and produces a dilution of the flux of CoRoT- 6 that has been corrected by subtracting the 2.8 percent of its median flux value. The flux inside the star's mask is split along detector column boundaries into broad-band red, green, and blue channels. To increase the signal-to-noise ratio and reduce systematic 
drifts present in individual channels, we sum up the flux in the red, green, and blue channels to obtain a light curve in a spectral range extending from 300 to $1100 \mathrm{~nm}$.

The observations and data processing are described by Fridlund et al. (2010), to whom we refer the reader for details. The reduction pipeline applies corrections for the sky background and the pointing jitter of the satellite, which is particularly relevant during ingress and egress from the Earth shadow. Measurements during the crossing of the South Atlantic Anomaly of the Earth's magnetic field, which amounts to about 7-9 percent of each satellite orbit, are discarded. Highfrequency spikes due to cosmic ray hits and outliers are removed by applying a 5-point running mean. The final duty cycle of the $32-\mathrm{s}$ observations is $\geq 90$ percent for the socalled N2 data time series that are accessible through the CoRoT Public Data Archive at IAS (http://idoc-corot. ias.u-psud. fr/). More information on the instrument, its operation, and performance can be found in Auvergne et al. (2009).

The transits are removed from the light curve using the ephemeris of Fridlund et al. (2010) and the out-of-transit data are binned by computing average flux values along each orbital period of the satellite (6184 s). This has the advantage of removing tiny systematic variations associated with the orbital motion of the satellite, still preserving the information on magneticinduced variability (cf. Alonso et al. 2008; Auvergne et al. 2009). In such a way, we obtain a light curve consisting of 1993 mean points ranging from HJD 2454572.5075 to 2454717.4519 , i.e., with a duration of 144.9443 days. The average standard error of the mean points is $2.41 \times 10^{-4}$ in relative flux units. We remove the long-term decrease of the flux, which may be due to the aging of the detector, by fitting a straight line to the data as in the case of CoRoT-4 (cf. Aigrain et al. 2008; Lanza et al. 2009b). The maximum flux in the de-trended binned time series at HJD 2454577.5245 is adopted as a reference unit level corresponding to the unspotted star, since the true value of the unspotted flux is unknown.

\section{Light curve modelling}

The reconstruction of the surface brightness distribution from the rotational modulation of the stellar flux is an ill-posed problem, because the variation of the flux vs. rotational phase contains only information on the distribution of the brightness inhomogeneities vs. longitude. The integration over the stellar disc effectively cancels any latitudinal information, particularly when the inclination of the rotation axis along the line of sight is close to $90^{\circ}$, as it is assumed in the present case (see Sect. 4 and Lanza et al. 2009a). Therefore, we need to include a priori information in the light curve inversion process to obtain a unique and stable map. This is done by computing a maximum entropy (hereafter $\mathrm{ME})$ map, which has been proven to successfully reproduce active region distributions and area variations in the case of the Sun (cf. Lanza et al. 2007). Our approach has been compared to other spot modelling methods by Mosser et al. (2009) and Huber et al. (2010). The occultations of the stellar disc by the planet during transits can be used to resolve the fine structure of the spot pattern and study its evolution (e.g., Silva-Valio et al. 2010). We plan to apply this approach to CoRoT-6 in a forthcoming paper (Silva-Valio et al., in prep.).

In our model, the star is subdivided into 200 surface elements, namely 200 squares of side $18^{\circ}$, with each element containing unperturbed photosphere, dark spots, and facular areas. The fraction of an element covered by dark spots is indicated by the filling factor $f$, the fractional area of the faculae is $Q f$, and the fractional area of the unperturbed photosphere is $1-(Q+1) f$. The contribution to the stellar flux coming from the $k$ th surface element at the time $t_{j}$, where $j=1, \ldots, N$ is an index numbering the $N$ points along the light curve, is given by:

$$
\begin{aligned}
\Delta F_{k j}= & I_{0}\left(\mu_{k j}\right)\left\{1-(Q+1) f+c_{\mathrm{s}} f\right. \\
& \left.+Q f\left[1+c_{\mathrm{f}}\left(1-\mu_{k j}\right)\right]\right\} A_{k} \mu_{k j} w\left(\mu_{k j}\right),
\end{aligned}
$$

where $I_{0}$ is the specific intensity in the continuum of the unperturbed photosphere at the isophotal wavelength of the observations, $c_{\mathrm{S}}$ and $c_{\mathrm{f}}$ are the spot and facular contrasts, respectively (cf. Lanza et al. 2004), $A_{k}$ is the area of the $k$ th surface element,

$w\left(\mu_{k j}\right)=\left\{\begin{array}{l}1 \text { if } \mu_{k j} \geq 0 \\ 0 \text { if } \mu_{k j}<0\end{array}\right.$

is its visibility, and

$\mu_{k j} \equiv \cos \psi_{k j}=\sin i \sin \theta_{k} \cos \left[\ell_{k}+\Omega\left(t_{j}-t_{0}\right)\right]+\cos i \cos \theta_{k}$,

is the cosine of the angle $\psi_{k j}$ between the normal to the surface element and the direction of the observer, with $i$ being the inclination of the stellar rotation axis along the line of sight, $\theta_{k}$ the colatitude and $\ell_{k}$ the longitude of the $k$ th surface element; $\Omega$ denotes the angular velocity of rotation of the $\operatorname{star}\left(\Omega \equiv 2 \pi / P_{\text {rot }}\right.$, with $P_{\text {rot }}$ the stellar rotation period), and $t_{0}$ the initial time. The specific intensity in the continuum varies according to a quadratic limb-darkening law, as adopted by Lanza et al. (2003) for the case of the Sun, viz. $I_{0} \propto a_{\mathrm{p}}+b_{\mathrm{p}} \mu+c_{\mathrm{p}} \mu^{2}$. The stellar flux computed at the time $t_{j}$ is then: $F\left(t_{j}\right)=\sum_{k} \Delta F_{k j}$. To warrant a relative precision of the order of $10^{-5}$ in the computation of the flux $F$, each surface element is further subdivided into $1^{\circ} \times 1^{\circ}$ elements and their contributions, calculated according to Eq. (1), are summed up at each given time to compute the contribution of the $18^{\circ} \times 18^{\circ}$ surface element to which they belong.

We fit the light curve by varying the value of $f$ over the surface of the star, while $Q$ is held constant. Even fixing the rotation period, the inclination, and the spot and facular contrasts (see Lanza et al. 2007, for details), the model has 200 free parameters and suffers from non-uniqueness and instability. To find a unique and stable spot map, we apply ME regularization by minimizing a functional $Z$, which is a linear combination of the $\chi^{2}$ and the entropy functional $S$; i.e.,

$Z=\chi^{2}(\boldsymbol{f})-\lambda S(\boldsymbol{f})$,

where $f$ is the vector of the filling factors of the surface elements, $\lambda>0$ a Lagrangian multiplier determining the trade-off between light curve fitting and regularization; the expression for $S$ is given in Lanza et al. (1998). The entropy functional $S$ attains its maximum value when the star is free of spots. Therefore, by increasing the Lagrangian multiplier $\lambda$, we increase the weight of $S$ in the model and the area of the spots is progressively reduced. This gives rise to systematically negative residuals between the observations and the best-fit model when $\lambda>0$. The optimal value of $\lambda$ depends on the information content of the light curve, which in turn depends on the ratio of the amplitude of its rotational modulation to the average errors of its points. In the case of CoRoT-6, the amplitude of the rotational modulation is $\sim 0.027$, while the average errors of the points is $\sim 2.4 \times 10^{-4}$ in relative flux units, giving a signal-to-noise ratio of $\sim 110$. This is sufficient to adopt the same regularization criterion applied in the case of CoRoT-2 (see Lanza et al. 2009a), iterating the value of $\lambda$ until the condition $\left|\mu_{\text {reg }}\right| \simeq \epsilon_{0}$ is met, where $\mu_{\text {reg }}$ is the mean of the residuals and $\epsilon_{0} \equiv \sigma_{0} / \sqrt{N}$ their standard 
error, i.e., the ratio of their standard deviation $\sigma_{0}$ in the case of the unregularized best fit (i.e., for $\lambda=0$ ) to the square root of the number $N$ of points in each fitted subset of the light curve having a duration $\Delta t_{\mathrm{f}}$ (see below).

In the case of the Sun, by assuming a fixed distribution of the filling factor, it is possible to obtain a good fit of the irradiance changes only for a limited time interval $\Delta t_{\mathrm{f}}$, not exceeding 14 days, which is the lifetime of the largest sunspot groups dominating the irradiance variation. In the case of other active stars, the value of $\Delta t_{\mathrm{f}}$ must be determined from the observations themselves, looking for the longest time interval that allows a good fit with the applied model (see Sect. 4).

The optimal values of the spot and facular contrasts and of the facular-to-spotted area ratio $Q$ in stellar active regions are unknown a priori. In our model the facular contrast $c_{\mathrm{f}}$ and the parameter $Q$ enter as the product $c_{\mathrm{f}} Q$, so we can fix $c_{\mathrm{f}}$ and vary $Q$, estimating its best value by minimizing the $\chi^{2}$ of the model, as shown in Sect. 4. Since the number of free parameters of the ME model is large, for this specific application we make use of the model of Lanza et al. (2003), which fits the light curve by assuming only three active regions to model the rotational modulation of the flux plus a uniformly distributed background to account for the variations of the mean light level. This procedure is the same used to fix the value of $Q$ in the cases of CoRoT-2, CoRoT-4, and CoRoT-7 (cf. Lanza et al. 2009a,b; Lanza et al. 2010).

We assume an inclination of the rotation axis of CoRoT-6 of $i \simeq 89^{\circ}$ in most of our models (see Sect. 4). Since the information on spot latitude that can be extracted from the rotational modulation of the flux for such a high inclination is negligible, the ME regularization virtually puts all the spots around the subobserver latitude (i.e., $90^{\circ}-i \approx 0^{\circ}$ ) to minimize their area and maximize the entropy. Therefore, we are limited to mapping only the distribution of the active regions vs. longitude, which can be done with a resolution better than $\sim 50^{\circ}$ (cf. Lanza et al. 2007, 2009a). Our ignorance of the true facular contribution may lead to systematic errors in the active region longitudes as derived by our model because faculae produce an increase of the flux when they are close to the limb, leading to a systematic shift of the longitudes of the active regions used to reproduce the observed flux modulation, as discussed by Lanza et al. (2007) for the case of the Sun and illustrated by Lanza et al. (2009a, cf. Figs. 4 and 5) for CoRoT-2.

\section{Model parameters}

The fundamental stellar parameters are taken from Fridlund et al. (2010) and are listed in Table 1. The limb-darkening parameters $a_{\mathrm{p}}, b_{\mathrm{p}}$, and $c_{\mathrm{p}}$ (cf. Sect. 3 ) have been derived from Kurucz (2000) model atmospheres for $T_{\text {eff }}=6090 \mathrm{~K}, \log g=$ $4.44\left(\mathrm{~cm} \mathrm{~s}^{-2}\right)$ and a metal abundance $[M / H]=-0.2$, by adopting the CoRoT white-band transmission profile given by Auvergne et al. (2009). The limb-darkening parameters $u_{+}=0.586 \pm 0.068$ and $u_{-}=-0.12 \pm 0.13$ obtained by fitting the transit cannot be used to constrain the above parameters because of their large errors (especially for $u_{-}$). However, they are compatible with those derived from Kurucz's models that yield $u_{+}=0.646$ and $u_{-}=-0.110$.

The rotation period adopted for our spot modelling has been derived from a Lomb-Scargle periodogram analysis of the light curve giving $P_{\text {rot }}=6.35 \pm 0.29$ days (Scargle 1982). The uncertainty of the period comes from the total extension of the time series and represents an upper limit. As we shall see below, our
Table 1. Parameters adopted for the light curve modelling of CoRoT-6.

\begin{tabular}{lrr}
\hline \hline Parameter & & Ref. \\
\hline Star Mass $\left(M_{\odot}\right)$ & 1.05 & F10 \\
Star Radius $\left(R_{\odot}\right)$ & 1.025 & F10 \\
$T_{\text {eff }}(\mathrm{K})$ & 6090 & F10 \\
$\log g\left(\mathrm{~cm} \mathrm{~s}^{-2}\right)$ & 4.44 & F10 \\
$a_{\mathrm{p}}$ & 0.340 & La10 \\
$b_{\mathrm{p}}$ & 1.024 & La10 \\
$c_{\mathrm{p}}$ & -0.378 & La10 \\
$P_{\text {rot }}$ (days) & 6.35 & La10 \\
$\epsilon$ & $1.71 \times 10^{-4}$ & La10 \\
Inclination $($ deg $)$ & 89.07 & F10 \\
$c_{\mathrm{s}}$ & 0.677 & La10 \\
$c_{\mathrm{f}}$ & 0.115 & L04 \\
$Q$ & 1.5 & La10 \\
$\Delta t_{\mathrm{f}}$ (days) & $6.15,7.247$ & La10 \\
\hline
\end{tabular}

References. F10: Fridlund et al. (2010); L04: Lanza et al. (2004); La10: present study.

models show that the starspots have a remarkable differential rotation, which may contribute to an uncertainty of the stellar rotation period of $\approx 10$ percent, i.e., of \pm 0.3 days, comparable with the above upper limit (cf. Sect. 5.2).

The polar flattening of the star due to the centrifugal potential is computed in the Roche approximation with a rotation period of 6.35 days. The relative difference between the equatorial and the polar radii $\epsilon$ is $1.71 \times 10^{-4}$ which induces a completely negligible relative flux variation of $\approx 10^{-6}$ for a spot coverage of $\sim 2$ percent as a consequence of the gravity darkening of the equatorial region of the star.

The inclination of the stellar rotation axis has not been constrained yet through the observation of the Rossiter-McLaughlin effect. Nevertheless, we assume that the stellar spin is normal to the orbital plane of the transiting planet, i.e., has an inclination of $89.07 \pm 0.30$ from the line of sight, unless otherwise specified. This is compatible with the measured $v \sin i=$ $8.0 \pm 1.0 \mathrm{~km} \mathrm{~s}^{-1}$, the above rotation period, and a radius estimate of $1.025 \pm 0.026 R_{\odot}$ from stellar evolutionary models, that yield $\sin i=0.98$ (cf. Fridlund et al. 2010). Taking into account their errors, the minimum inclination still compatible with those stellar parameters is $\sim 50^{\circ}$.

The maximum time interval that our model can accurately fit with a fixed distribution of active regions $\Delta t_{\mathrm{f}}$ has been determined by dividing the total interval, $T=144.9443$ days, into $N_{\mathrm{f}}$ equal segments, i.e., $\Delta t_{\mathrm{f}}=T / N_{\mathrm{f}}$, and looking for the minimum value of $N_{\mathrm{f}}$ that allows us a good fit of the light curve, as measured by the $\chi^{2}$ statistics. We find that for $N_{\mathrm{f}}<20$ the quality of the best fit degrades significantly with respect to $N_{\mathrm{f}} \geq 20$, owing to a substantial evolution of the pattern of surface brightness inhomogeneities. Therefore, we adopt $\Delta t_{\mathrm{f}}=7.2472$ days as the maximum time interval to be fitted with a fixed distribution of surface active regions in order to estimate the best value of the parameter $Q$ (see below). For the spot modelling in Sects. 5.1 and 5.2, we shall consider the light curve interval from HJD 2454576.0 to 2454717.4519 because the light modulation is not well fitted during the first three days owing to the rapid evolution of some active regions (see Sect. 5.1), and adopt $N=23$ corresponding to $\Delta t_{\mathrm{f}}=6.15$ days, which provides a better time resolution to study the evolution of the spot pattern during the intervals with faster modifications.

To compute the spot contrast, we adopt the same mean temperature difference as derived for sunspot groups from their 


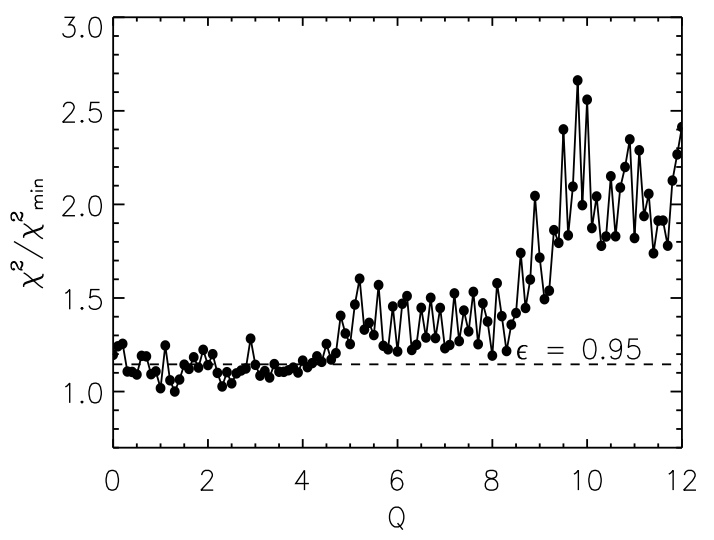

Fig. 1. The ratio of the $\chi^{2}$ of the composite best fit of the entire time series (from HJD 2454572.5075 to 2454717.4519 ) to its minimum value vs. the parameter $Q$, i.e., the ratio of the facular area to the cool spot area in active regions. The horizontal dashed line indicates the 95 percent confidence level for $\chi^{2} / \chi_{\min }^{2}$, determining the interval of acceptable $Q$ values.

bolometric contrast, i.e., $560 \mathrm{~K}$ (Chapman et al. 1994). In other words, we assume a spot effective temperature of $5530 \mathrm{~K}$, yielding a contrast $c_{\mathrm{s}}=0.677$ in the CoRoT passband (cf. Lanza et al. 2007). A different spot contrast changes the absolute spot coverages, but does not significantly affect their longitudes and their time evolution, as discussed in detail by Lanza et al. (2009a). The facular contrast is assumed to be solar-like with $c_{\mathrm{f}}=0.115$ (Lanza et al. 2004).

The best value of the area ratio $Q$ of the faculae to the spots in each active region has been estimated by means of the model of Lanza et al. (2003, cf. Sect. 3). In Fig. 1, we plot the ratio $\chi^{2} / \chi_{\min }^{2}$ of the total $\chi^{2}$ of the composite best fit of the entire time series to its minimum value $\chi_{\min }^{2}$, versus $Q$, and indicate the 95 percent confidence level as derived from the F-statistics (e.g., Lampton et al. 1976). Choosing $\Delta t_{\mathrm{f}}=7.2472$ days, we fit the rotational modulation of the active regions for the longest time interval during which they remain stable, modelling both the flux increase due to the facular component when an active region is close to the limb as well as the flux decrease due to the dark spots when the same region transits across the central meridian of the disc. In such a way, a measure of the relative facular and spot contributions can be obtained leading to a fairly accurate estimate of $Q$.

The best value of $Q$ turns out to be $Q \simeq 1.5$, with an acceptable range extending from 0 to $\sim 4$. The $\chi^{2}$ fluctuates owing to the numerical errors occurring during its minimization when a given best fit is computed. Therefore, it is its overall variation versus $Q$ that is relevant here. In the case of the Sun, the best value of $Q$ is 9 (Lanza et al. 2007). Thus our result indicates a lower relative contribution of the faculae to the light variation of CoRoT-6 than in the solar case. The relative amplitude of the rotational modulation of the star is $\sim 0.027$, showing that CoRoT-6 is more active than the Sun, for which a relative variation of $\sim 0.0035$ is observed at the maximum of the 11-yr cycle. This may explain the reduced facular contribution to the light variations of CoRoT-6, as suggested by Radick et al. (1998) and Lockwood et al. (2007) who find a smaller facular signature in stars more active than the Sun.

The use of the chromatic information of the CoRoT light curves to estimate the spot and facular contrasts and filling factors is made impossible by our ignorance of the unperturbed stellar flux levels in the different colour channels which are needed

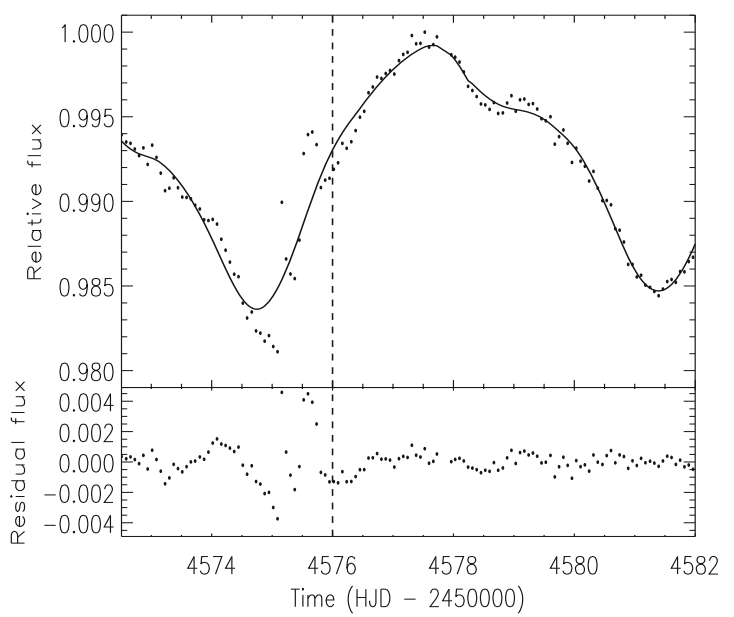

Fig. 2. Upper panel: the out-of-transit light curve of CoRoT-6 (dots) in the time interval between HJD 2454572.5075 and 2454582.0 and its unregularized best fit for a facular-to-spotted area ratio of $Q=1.5$ (solid line). The flux is normalized to the maximum at HJD 2454577.5245. Lower panel: the corresponding residuals. Note the greater residuals before HJD 2454576.0 (marked by the vertical dashed line) owing to the rapid evolution of some active regions.

to disentangle the flux perturbations due to spots and faculae, respectively. The continuous variations of the observed fluxes do not allow us to fix such reference levels so that we cannot unambiguously attribute a given flux modulation to cool spots or bright faculae. Moreover, the oscillations of the fluxes in the individual colour channels on timescales comparable with the rotation period and the lack of precise passband profiles, make the extraction of information on spots and faculae unfeasible.

\section{Results}

\subsection{Light curve model}

We initially apply the model of Sect. 3 to the whole out-of-transit CoRoT-6 light curve. Even without any regularization, the fit to the time interval between HJD 2454572.5075 and 2454576.0 is not satisfactory owing to the remarkable flux variation on a timescale of $\sim 2$ days associated with the rapid evolution of some active regions (cf. Fig. 2). It cannot be adequately fitted by our model which assumes that the pattern of active regions stays constant during the time interval $\Delta t_{\mathrm{f}}>2$ days. Therefore, we decide to discard the first interval of the light curve, the endpoint of which is marked by the vertical dashed line in Fig. 2, and analyse the section from HJD 2454576.0 to 2454717.4519 covering 141.4519 days. We consider 23 individual intervals of $\Delta t_{\mathrm{f}}=6.15$ days which warrant a good fitting and an adequate time resolution to trace the evolution of the active regions.

The best fit without regularization $(\lambda=0)$ has a mean $\mu_{\text {res }}=5.739 \times 10^{-6}$ and a standard deviation of the residuals $\sigma_{0}=4.155 \times 10^{-4}$ in relative flux units which is 1.7 times the standard error of the mean points. This is due to the flux fluctuations on a timescale of $\sim 2$ days associated with a populations of short-lived active regions that can be only partially fitted by our approach (see below).

The Lagrangian multiplier $\lambda$ is iteratively adjusted until the mean of the residuals $\mu_{\text {res }}=-4.962 \times 10^{-5} \simeq-\sigma_{0} / \sqrt{N}$, where $N=84$ is the mean number of points in each fitted light curve interval $\Delta t_{\mathrm{f}}$. The standard deviation of the residuals of 


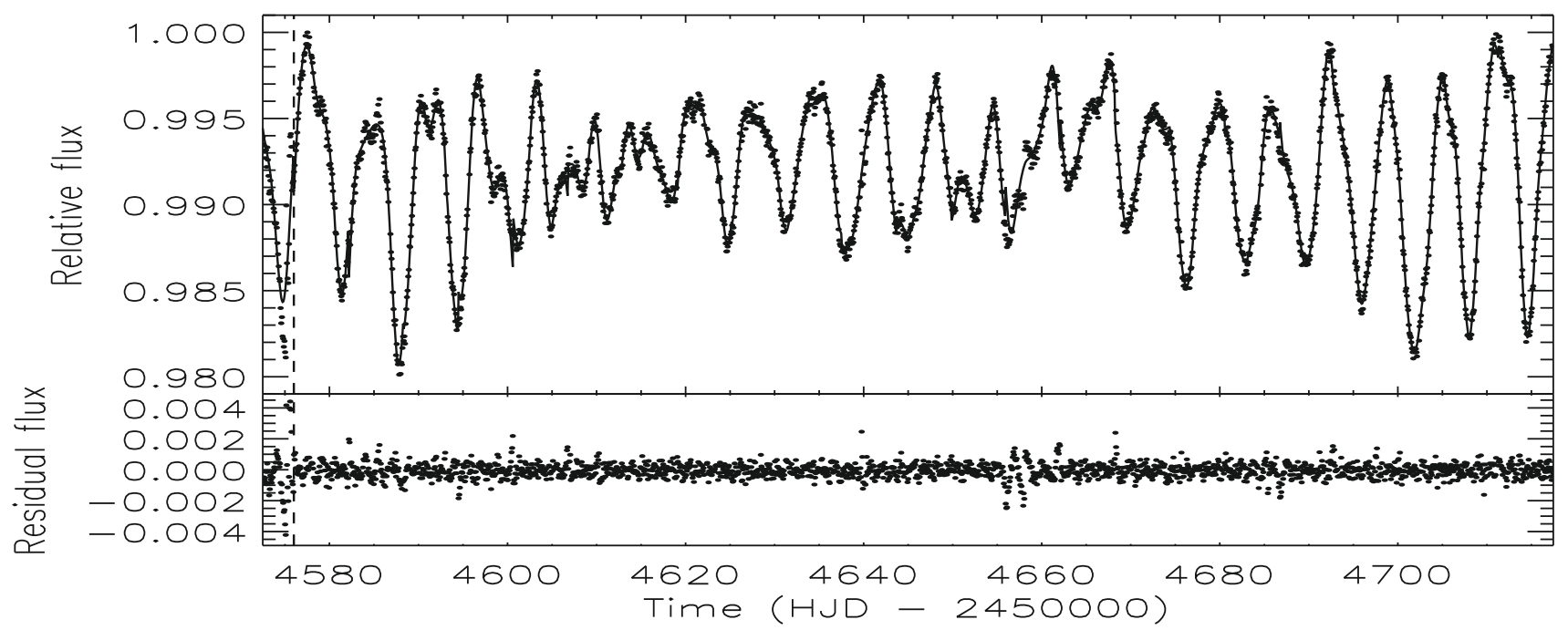

Fig. 3. Upper panel: the out-of-transit light curve of CoRoT-6 (dots) and its ME-regularized best fit for a facular-to-spotted area ratio of $Q=1.5$ (solid line) during the time interval between HJD 2454572.5075 and 2454717.4519. The flux is normalized to the maximum observed flux. Lower panel: the corresponding residuals. In both panels, the vertical dashed line marks HJD 2454576, i.e., the beginning of the interval considered for the modelling (see the text).

the regularized best fit is $\sigma=4.728 \times 10^{-4}$. The composite best fit to the entire light curve is shown in the upper panel of Fig. 3 while the residuals are plotted in the lower panel. The fit is generally good, although the residuals sometimes show significant excursions to be attributed to the rapidly evolving active regions with lifetimes of $1-3$ days that cannot be fitted by our approach. In particular, during the first part of the observations up to HJD 2454576 marked by the vertical dashed line, the residuals are a factor of $\sim 4-5$ larger than during most of the subsequent interval. A ME model computed from HJD 2454576.0 to 2454717.4519 with $Q=4$ shows a marginally better fit with an average $\sigma=4.572 \times 10^{-4}$. Nevertheless, we decide to adopt $Q=1.5$ in our analysis, corresponding to the minimum of the total $\chi^{2}$ of the fit obtained with the three-spot model, as described in Sect. 4.

\subsection{Longitude distribution of active regions and stellar differential rotation}

The distribution of the spot covering factor $f$ versus longitude and time is plotted in Fig. 4 for our ME models with $Q=1.5$. The origin of the longitude corresponds to the sub-observer point at the epoch HJD 2454576.0 and the longitude increases in the same direction as the stellar rotation and the orbital motion of the planet. The lifetimes of the mapped active regions range from $\sim 6$ days, that is the time resolution of our spot modelling, to $\sim 30-40$ days, as can be inferred from the isocontours of their filling factors in Fig. 4. Most of the active regions grow and decay within five active longitudes that can be identified by their uniform migration in the plot. Although there is always some degree of arbitrareness in attributing a given active region to a particular active longitude, we trace the migration of these longitudes by white dashed lines. Their migration rates range from $-3.88 \pm 0.75 \mathrm{deg} /$ day to $3.13 \pm 0.75 \mathrm{deg} / \mathrm{day}$. If such different rates correspond to the angular velocity at different latitudes on a differentially rotating star, the relative amplitude of the differential rotation is estimated to be $\Delta \Omega / \Omega=0.123 \pm 0.020$. This is actually a lower limit for the pole-equator angular velocity difference because we do not have information on the latitude of the active regions which are probably confined to some range as

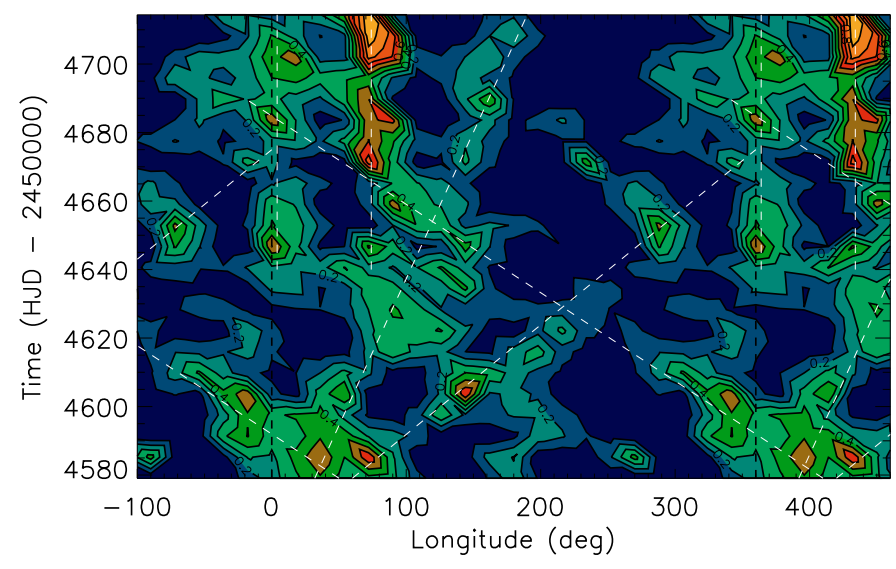

Fig. 4. Isocontours of the ratio $f / f_{\max }$, where $f$ is the spot covering factor and $f_{\max }=0.0059$ its maximum value, versus time and longitude for the ME models with $Q=1.5$. The two dashed black lines mark longitudes $0^{\circ}$ and $360^{\circ}$ beyond which the distributions are repeated to easily follow spot migration. The contour levels are separated by $\Delta f=$ $0.1 f_{\max }$ with yellow indicating the maximum covering factor and dark blue the minimum. The dashed white lines trace the migration of the active regions associated with each active longitude (see the text).

we observe in the Sun where spots appear only in the $\pm 40^{\circ}$ belt. A consequence of this interpretation is that the intersections between the straight lines tracing the migration of different active longitudes in Fig. 4 do not necessarily imply a coincidence of the corresponding active regions because they are probably located at different latitudes.

A ME spot model computed with $Q=4$ leads to a somewhat smaller differential rotation, i.e., $\Delta \Omega / \Omega \simeq 0.09 \pm 0.02$, because the reconstructed longitudes of the active regions are more affected by the flux modulation due to their facular component (cf. Sect. 3). Reducing the inclination $i$ of the stellar rotation axis to $70^{\circ}$ does not significantly change the amplitude of the differential rotation. On the other hand, for $i=50^{\circ}$, several small spots are ill-mapped making it difficult to trace the migration of the active longitudes rotating faster than the mean period of 


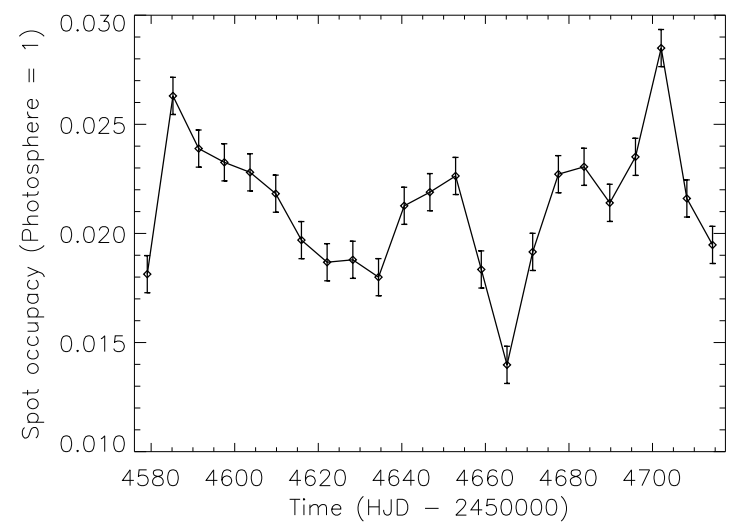

Fig. 5. The total spotted area vs. time, as derived from our lightcurve model illustrated in Figs. 3 and 4 . The uncertainty of the area ( $3 \sigma$ errorbars) has been derived from the standard deviation of the residuals of the best fit to the light curve.

6.35 days. This leads to an estimated $\Delta \Omega / \Omega$ about half the above value.

The amplitude of the surface differential rotation of CoRoT-6 is in agreement with the mean value found for stars of comparable effective temperature and rotation period by fitting the shape of their line profiles or by Doppler imaging techniques (Barnes et al. 2005; Reiners 2006). For CoRoT-4, that has a slightly hotter effective temperature of $6190 \mathrm{~K}$ and a mean rotation period of $\sim 9$ days, we estimate a lower limit for the surface differential rotation $\Delta \Omega / \Omega=0.057 \pm 0.015$ from the ME spot models with $Q=4.5$ (Lanza et al. 2009b). It may be explained by assuming that the active regions mapped on that star are closer to the equator. By comparison, the relative angular velocity difference in the $\pm 40^{\circ}$ sunspot belt amounts to $\sim 0.05$.

The total spotted area as derived from our modelling is plotted vs. time in Fig. 5. Since we removed the long-term linear trend in the flux, the associated variation of the spotted area is not included in our model. There is no clear periodicity in the variation, although timescales ranging from $\sim 25$ to $\sim 60$ days are apparent.

\subsection{Signatures of a possible star-planet interaction}

In the reference frame adopted for our spot modelling, which rotates with a period $P_{\text {rot }}=6.35$ days with respect to an inertial frame, the period between subsequent crossings of a given meridian by the subplanetary point is the synodic period $P_{\text {syn }}=$ 22.25 days, defined as $P_{\text {syn }}^{-1}=P_{\text {rot }}^{-1}-P_{\text {orb }}^{-1}$, where the orbital period of the planet $P_{\text {orb }}=8.886$ days.

Considering the distribution of the spot filling factor $f$ vs. time and longitude, we find a correlation of the longitude of some active regions with the meridian located at $\Delta \ell=-200^{\circ}$ with respect to the subplanetary longitude (cf. Fig. 6). The white lines overplotted on the distribution of $f$ show the motion of such a meridian vs. time with the period $P_{\text {syn. }}$. The active regions that reach a maximum filling factor close to their passage across this meridian are marked with crosses in Fig. 6 and listed in Table 2, where the columns from the left to the right report the time of their maximum filling factor, their longitude $\ell_{\text {ar }}$ at that time, the filling factor per $18^{\circ}$ longitude bin $f / f_{\max }$, normalized to the maximum filling factor over the whole map $\left(f_{\max }=0.0059\right)$, the distance $\delta \ell$ of the active region from the active meridian, and

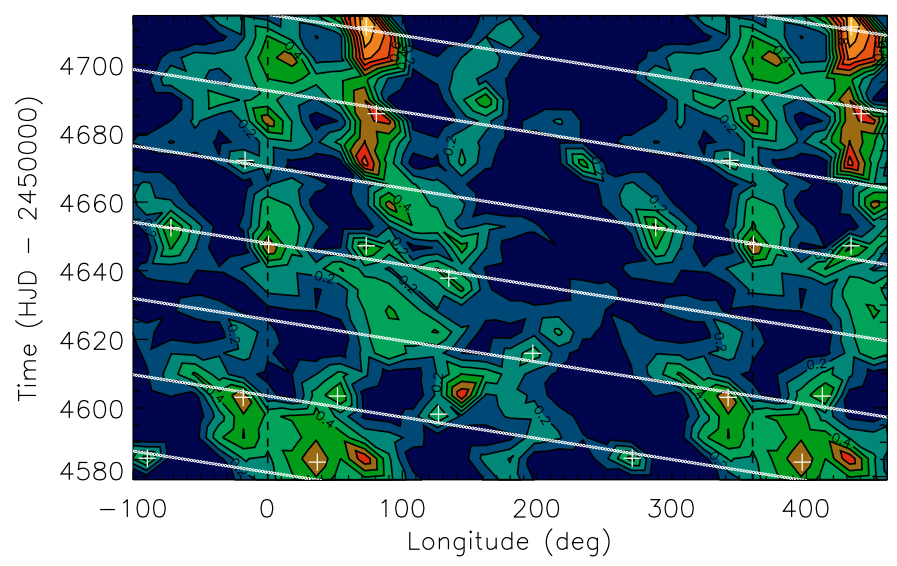

Fig. 6. Isocontours of the spot filling factor as in Fig. 4 with white lines tracing the active meridian lagging the subplanetary meridian by $\Delta \ell=$ $-200^{\circ}$. White crosses indicate the relative maxima of the spot filling factor corresponding to the active regions listed in Table 2.

Table 2. Stellar active regions associated with the active meridian.

\begin{tabular}{crcrc}
\hline \hline $\begin{array}{c}\text { Time } \\
\text { (HJD-2450000) }\end{array}$ & $\begin{array}{r}\ell_{\text {ar }} \\
(\mathrm{deg})\end{array}$ & $f / f_{\max }$ & $\begin{array}{r}\delta \ell \\
(\mathrm{deg})\end{array}$ & $p$ \\
\hline 4583.68 & 36.2 & 0.563 & 75.1 & 1.000 \\
4584.81 & 270.0 & 0.417 & 32.8 & 0.918 \\
4597.65 & 126.2 & 0.387 & 31.2 & 0.873 \\
4602.56 & 341.2 & 0.184 & 34.4 & 0.964 \\
4602.93 & 51.2 & 0.452 & 41.7 & 1.000 \\
4615.39 & 196.2 & 0.327 & 28.3 & 0.792 \\
4637.29 & 133.8 & 0.328 & 39.9 & 1.000 \\
4646.73 & 72.5 & 0.515 & 51.6 & 1.000 \\
4647.10 & 0.0 & 0.591 & 14.8 & 0.414 \\
4652.01 & 287.5 & 0.505 & 7.9 & 0.220 \\
4671.64 & 342.5 & 0.335 & 4.8 & 0.135 \\
4685.23 & 80.0 & 0.543 & 37.8 & 1.000 \\
4710.53 & 72.5 & 0.965 & 4.0 & 0.113 \\
\hline
\end{tabular}

the probability $p$ of a chance association between them, respectively. The latter is computed according to:

$p=\min \left\{n_{\mathrm{ar}} \frac{\delta \ell}{\Delta L}, 1\right\}$,

where $n_{\mathrm{ar}}$ is the number of active regions appearing during a given synodic period and $\Delta L$ the longitude range where stellar activity is mainly localized. If activity were distributed uniformly, $\Delta L=360^{\circ}$, but in CoRoT- 6 most of the active regions appear within a longitude range $\Delta L \sim 250^{\circ}$, owing to the presence of active longitudes. Therefore, we adopt $\Delta L=250^{\circ}$ and $n_{\mathrm{ar}}=7$ to compute the probabilities in the last column of Table 2 . The adopted $n_{\mathrm{ar}}$ corresponds to the maximum number of active regions observed in one synodic period, thus yielding the maximum probability of chance association.

The total probability $p_{\mathrm{T}}$ of a chance occurrence for the entire set of 13 active regions is:

$p_{\mathrm{T}}=\frac{\prod_{i} p_{i}}{\min \left\{p_{i}\right\}}$.

The division by $\min \left\{p_{i}\right\}$ accounts for the additional degree of freedom related to the fact that the phase lag $\Delta \ell=-200^{\circ}$ 


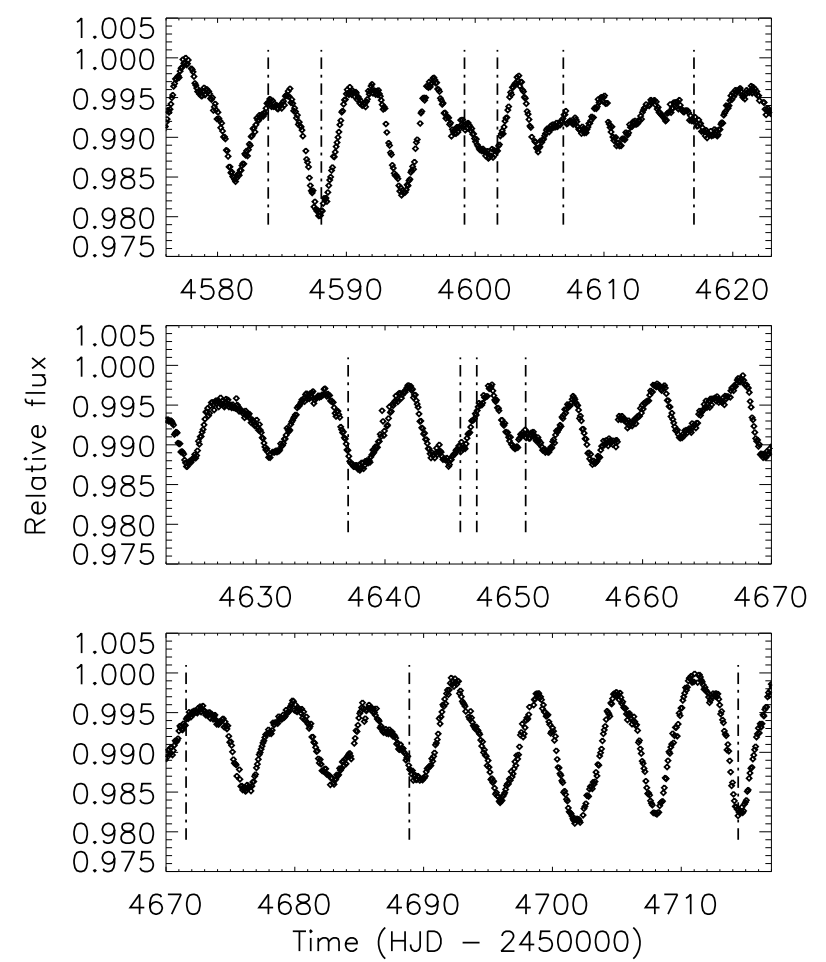

Fig. 7. The light curve of CoRoT-6 with the epochs of maximum visibility of the active regions listed in Table 2 marked by vertical dash-dotted lines.

between the active and the subplanetary meridians is not fixed a priori but is derived from the same set of data. In such a way, we obtain a probability of chance occurrence $p_{\mathrm{T}}=0.0075$, indicating that the association of those active regions with the active meridian is significant.

Spot models with a greater facular contribution, i.e., $Q=$ 4.0 , confirm the presence of active regions associated with the orbital motion of the planet, although $\Delta \ell=-170^{\circ}$, owing to the systematic longitude shifts of the active regions associated with a greater value of $Q$.

Since it is not possible to exclude a misalignment between the stellar spin and the orbital angular momentum, we have investigated the impact of a different inclination of the stellar rotation axis on the above correlation finding that the active regions listed in Table 2 are well reproduced also when $i=70^{\circ}$. On the other hand, only one third of them survive when $i=50^{\circ}$, which corresponds to the minimum inclination compatible with the estimated stellar parameters (cf. Sect. 4). In this case, no significant evidence of star-planet interaction is found.

In Fig. 7 we plot the light curve of CoRoT-6 and mark the epochs corresponding to the passages of the active regions listed in Table 2 across the central meridian of the stellar disc. The two remarkable active regions reaching the maximum filling factor at HJD 2454583.68 and 2454710.53 correspond to flux minima at HJD 2454588.1 and 2454714.4, respectively, while the other smaller active regions are generally associated with changes of the slope of the light curve. Such slope changes can be attributed to the variation of their area (or filling factor) because the projection factor is constant when an active region crosses the central meridian.

The separations between the vertical marks in Fig. 7 depend on the epochs of maximum visibility of the corresponding active regions, which coincide with the times when they cross the central meridian of the disc and are in general not simultaneous with the maxima of their filling factors as listed in Table 2. Specifically, given that the rotation period of our reference frame is $P_{\text {rot }}=6.35$ days, the mark corresponding to a given active region in Fig. 7 is shifted from the epoch of its maximum filling factor by a time interval depending on its longitude that can range up to $\pm P_{\text {rot }}$. Their different longitudes and the fact that the active regions associated with the star-planet interaction do not appear periodically, lead to an uneven distribution of the corresponding marks vs. time in Fig. 7.

\section{Discussion}

CoRoT-6 is an interesting system to study possible interactions between the planet and its host star because its rotation is not synchronized with the planetary orbit allowing us to separate the rotational modulation from a signal at the orbital or the synodic period. For comparison, CoRoT-4 is a slightly hotter star, but it is synchronized, thus an activity signature of planetary origin is more difficult to find out. Nevertheless, a persistent active region close to the subplanetary longitude has been observed in that star during the $\sim 70$ days of CoRoT observations (Lanza et al. 2009b). Another interesting case is that of $\tau$ Bootis, also synchronized to the orbital motion of its hot Jupiter. In this star, Walker et al. (2008) found evidence of a persistent photospheric active region leading the subplanetary longitude by $\approx 70^{\circ}$.

The other remarkably active star CoRoT- 2 shows a shortterm activity cycle with a period of $\sim 28.9$ days, as indicated by the variation of the total area of its spots, that is very close to ten synodic periods of the planet with respect to the mean stellar rotation period of 4.52 days. As a matter of fact, the synodic period of CoRoT-2 is only 2.84 days, which is shorter than the time resolution $\Delta t_{\mathrm{f}}=3.16$ days of the spot mapping performed by Lanza et al. (2009a) making it impossible to look for the effects found in CoRoT-6 whose synodic period is 22.25 days, i.e., remarkably longer than the time resolution of our mapping $\left(\Delta t_{\mathrm{f}}=6.15\right.$ days). However, Pagano et al. (2009) find a systematic increase of the variance of the optical flux immediately before the planetary transits in the light curve of CoRoT- 2 which suggests short-lived active regions or flaring activity close to the subplanetary longitude.

A major difficulty with the allegedly cases of magnetic starplanet interactions comes from the remarkably different behaviours observed in different stars that makes it impossible to define a common and simple phenomenology. Moreover, in the case of CoRoT-6, there is no stable periodicity in the emergence of the active regions allegedly associated with the interaction and this makes the proposed correlation true only in a statistical sense. The lifetimes of the active regions listed in Table 2 are comparable with the synodic period, thus, sooner or later, they should cross the straight lines marking the active meridian in Fig. 6 during their migration at the rates characteristic of the active longitudes to which they belong. Therefore, what suggests the presence of a star-planet interaction is not just the coincidence in longitude, but the fact that the maximum filling factors of those active regions are reached very close to such a crossing.

This can be interpreted as the result of some triggering of activity associated with the passage of the active meridian over a previously existing active region, possibly due to an enhancement of the rate of magnetic flux emergence from the subphotospheric layers where magnetic flux is stored. In principle, two possible mechanisms can be invoked to explain this effect, respectively associated with the tidal force or with the magnetic field perturbation in the stellar corona. 
Tidal effects in CoRoT-6 are very weak because the semimajor axis of the planetary orbit is 17.9 times the radius of the star (Fridlund et al. 2010). Specifically, the tidal synchronization time for the star is $\sim 900 \mathrm{Gyr}$ for a tidal quality factor $Q^{\prime}=10^{6}$, i.e., much longer than the estimated stellar age ranging between 1 and 3.3 Gyr (Fridlund et al. 2010). Moreover, tidal effects are unlikely to be responsible for the observed star-planet interaction because they would produce two maxima of activity on opposite hemispheres associated with the two opposite tidal bulges instead of the suggested intermittent activity associated with a meridian rotating with the synodic period.

A perturbation of the hydromagnetic dynamo in the subsurface layers of a star induced by a close-in planet has been considered by Lanza (2008). The conjectured process assumes that the reconnection occurring in the stellar corona between the magnetic fields of the star and the planet generates a perturbation of the magnetic helicity that is conducted down to the stellar surface and immediately below it along magnetic field lines. The longitude where the perturbation of the dynamo action is maximum coincides with the footpoint of the magnetic field line connecting the surface of the star to the planet. If the stellar dynamo is not axisymmetric, when an active longitude crosses that footpoint the magnetic field in the subphotospheric layers can be intensified locally, giving rise to the observed maximum of activity in the active region. The phenomenon is not periodic because the emergence of the magnetic flux requires that the intensity of the unperturbed field be already close to the threshold for the buoyancy instability and this occurs only where there is a pre-existing active region. Since active regions appear in a non-periodic fashion and are preferentially located within active longitudes migrating at different rates, there is no well-defined periodicity in the star-planet interaction, but only some correlation with the activity already found in the active longitudes.

In the model of Lanza (2008), the coronal magnetic field has a non-potential configuration so its field lines are twisted and this can account for the observed lag $\Delta \ell$ between the active and the subplanetary meridians. Lanza (2009) proposes that the minimum energy configuration of the stellar coronal field is a linear force-free state, i.e., with the field satisfying the equation $\nabla \times \boldsymbol{B}=\alpha \boldsymbol{B}$, where $\alpha$ is uniform. In principle, a unique linear force-free configuration can be found when the magnetic field components are given at the photosphere and the angle of twist of the field lines between the surface of the star and the orbit of the planet is specified. Unfortunately, in our case we have only information on the latter quantity, so there are potentially infinite configurations leading to the observed phase lag between the active longitude and the subplanetary point. For a phase lag $\Delta \ell=-200^{\circ}$, one of the possible configurations is obtained for $\alpha=0.085$, while the other field parameters specifying the variation of the field strength with radius have been fixed at the values $b_{0}=-1.1$ and $c_{0}=1.0$, as adopted by Lanza (2008).

The topology of the coronal magnetic field plays an important role in the angular momentum loss of the star through its magnetized wind. The interaction between the planet and the coronal field could modify this topology leading to a reduction of the braking rate by $\approx 30$ percent (see Lanza 2010, for details). Indeed, the rotation period of CoRoT-6 seems to be quite short for a star of its mass and a minimum estimated age of $\approx 1$ Gyr (Fridlund et al. 2010). Specifically, the gyrochronology relationship of Barnes (2009) gives a period of $\sim 9$ days, i.e., about 30 percent longer than observed for a star of that mass and age. The difference is significant because the age spread allowed for $P_{\text {rot }} \sim 6-7$ days is about \pm 100 Myr for a predicted age of 500 Myr. On the other hand, it is not possible to exclude that the star is remarkably younger than 1 Gyr because this lower limit was estimated from the Lithium abundance which has a weak dependence on the stellar age in that range of mass. Of course, a younger age $(\approx 400-600 \mathrm{Myr})$ would reconcile gyrochronology with the measured rotation period. In the case of CoRoT-6, tidal effects on the evolution of the stellar spin are unlikely, given the very weak tidal force exerted by the planet that moves on a relatively wide orbit.

Finally, we note that possible signatures of the star-planet interaction can be searched for also in the modulation of the chromospheric line fluxes (e.g. Shkolnik et al. 2005, 2008). From the correlation among chromospheric emission, spectral type and rotation found by Noyes et al. (1984), a residual flux in the core of the Ca II H\&K lines $R_{\mathrm{HK}}^{\prime} \approx 2.8$ that of the Sun can be estimated (Foing, priv. comm.). It could be observable if high resolution $(\gtrsim 60000)$ and sufficiently high signal-to-noise ratio $(S / N \gtrsim 30-40)$ spectra of the Ca II H\&K region are available (cf., e.g., Rebolo et al. 1989). Since the $v \sin i$ of CoRoT-6 is $7.5 \pm 1.0 \mathrm{~km} \mathrm{~s}^{-1}$, it could be possible to derive the longitudes of the chromospheric plages by, e.g., the method proposed by Char \& Foing (1993), and study their possible relationship with the planetary orbital phase.

\section{Conclusions}

We have applied a ME spot model to fit the optical light curve of the active planet-hosting star CoRoT- 6 deriving maps of the distributions of its active regions vs. longitude with a time resolution of 6.15 days during 141 days of uninterrupted CoRoT observations. Active regions are assumed to consist of cool spots and bright faculae with solar-like contrasts. The facular-to-spotted area ratio in each active region is assumed to be constant and the best value is found to be $Q=1.5$ with an acceptable range between 0 and $\sim 4$. This is typical of a star with an activity level higher than the Sun.

Our maps show five active longitudes where individual active regions preferentially grow and decay with typical lifetimes up to $\sim 30-40$ days. The migration of the active longitudes allows us to derive a lower limit for the surface differential rotation $\Delta \Omega / \Omega=0.12 \pm 0.02$.

We find several active regions reaching a maximum of activity at a longitude lagging the subplanetary meridian by $\Delta \ell \sim$ $-200^{\circ}$. A spurious coincidence, owing to a chance appearance of those active regions, can be excluded with a degree of confidence greater than 99 percent. This suggests the presence of some magnetic star-planet interaction, although the correlation we found is significant only in a statistical sense.

Acknowledgements. The authors are grateful to the Referee, Prof. Gordon Walker, for a careful reading of the manuscript and valuable comments, and to Dr. Bernard Foing for interesting discussions. Active star research and exoplanetary studies at INAF-Osservatorio Astrofisico di Catania and Dipartimento di Fisica e Astronomia dell'Università degli Studi di Catania are funded by MIUR (Ministero dell'Istruzione, dell'Università e della Ricerca) and by Regione Siciliana, whose financial support is gratefully acknowledged. This research has made use of the CoRoT Public Data Archive operated at IAS, Orsay, France, and of the ADS-CDS databases, operated at the CDS, Strasbourg, France.

\section{References}

Aigrain, S., Collier Cameron, A., Ollivier, M., et al. 2008, A\&A, 488, L43 Alonso, R., Auvergne, M., Baglin, A., et al. 2008, A\&A, 482, L21 Auvergne, M., Bodin, P., Boisnard, L., et al. 2009, A\&A, 506, 411 
A. F. Lanza et al.: Activity, rotation, and star-planet interaction of CoRoT-6

Baglin, A., Ackermann, M., Boisnard, L., et al. 2006, 36th COSPAR Scientific Assembly, 36, 3749

Barnes, S. 2009, IAU Symp., 258, 345

Barnes, J. R., Cameron, A. C., Donati, J.-F., et al. 2005, MNRAS, 357, L1

Chapman, G. A., Cookson, A. M., \& Dobias, J. J. 1994, ApJ, 432, 403

Char, S., \& Foing, B. H. 1993, A\&A, 276, 69

Fridlund, M., Hébrard, G., Alonso, R., et al. 2010, A\&A, 512, A14

Huber, K. F., Czesla, S., Wolter, U., \& Schmitt, J. H. M. M. 2010, A\&A, 514, A39

Kurucz, R. L. 2000, http: //kurucz . harvard.edu/

Lampton, M., Margon, B., \& Bowyer, S. 1976, ApJ, 208, 177

Lanza, A. F. 2008, A\&A, 487, 1163

Lanza, A. F. 2009, A\&A, 505, 339

Lanza, A. F. 2010, A\&A, 512, A77

Lanza, A. F., Catalano, S., Cutispoto, G., Pagano, I., \& Rodonò, M. 1998, A\&A, 332,541

Lanza, A. F., Rodonò, M., Pagano, I., Barge P., \& Llebaria, A. 2003, A\&A, 403, 1135

Lanza, A. F., Rodonò, M., \& Pagano, I. 2004, A\&A, 425, 707

Lanza, A. F., Bonomo, A. S., \& Rodonò, M. 2007, A\&A, 464, 741
Lanza, A. F., Pagano, I., Leto, G., et al. 2009a, A\&A, 493, 193

Lanza, A. F., Aigrain, S., Messina, S., et al. 2009, A\&A, 506, 255

Lanza, A. F., Bonomo, A. S., Moutou, C., et al. 2010, A\&A, 520, A53

Lockwood, G. W., Skiff, B. A., Henry, G. W., et al. 2007, ApJS, 171, 260

Mosser, B., Baudin, F., Lanza, A. F., et al. 2009, A\&A, 506, 245

Noyes, R. W., Hartmann, L. W., Baliunas, S. L., Duncan, D. K., \& Vaughan, A. H. 1984, ApJ, 279, 763

Pagano, I., Lanza, A. F., Leto, G., et al. 2009, Earth Moon and Planets, 105, 373

Radick, R. R., Lockwood, G. W., Skiff, B. A., \& Baliunas, S. L. 1998, ApJS, 118, 239

Rebolo, R., Garcia Lopez, R., Beckman, J. E., et al. 1989, A\&AS, 80, 135

Reiners A. 2006, A\&A, 446, 267

Scargle, J. D. 1982, ApJ, 263, 835

Shkolnik, E., Walker, G. A. H., Bohlender, D. A., Gu, P.-G., \& Kürster, M. 2005, ApJ, 622, 1075

Shkolnik, E., Bohlender, D. A., Walker, G. A. H., \& Collier Cameron, A. 2008, ApJ, 676, 628

Shkolnik, E., Aigrain, S., Crammer, S., et al. 2009, AIP Conf. Ser., 1094, 275

Silva-Valio, A., Lanza, A. F., Alonso, R., \& Barge, P. 2010, A\&A, 510, A25

Walker, G. A. H., Croll, B., Matthews, J. M., et al. 2008, A\&A, 482, 691 